\title{
Management of mental health, job professionalism and job satisfaction in junior gynecologists
}

\author{
Tomoyuki Kawada ${ }^{1}$
}

Received: 23 January 2017 / Accepted: 13 February 2017 / Published online: 1 March 2017

(C) Springer-Verlag Berlin Heidelberg 2017

I read with great interest the article titled "Being prepared to work in Gynecology Medicine: evaluation of an intervention to promote junior gynecologists professionalism, mental health and job satisfaction" by Mache et al. [1]. The authors conducted an intervention study on junior gynecologists' psychosocial skills for 12 weekly sessions. Training content involved developing and learning coping skills as well as solution-focused and cognitive behavioral counselling. Study outcomes were perceived occupational stress, emotional exhaustion, resilience coping behavior, emotion regulation skills and job satisfaction. Surveys were conducted four times in total at baseline, after the training, after 3 and 6 months. Almost all the outcomes significantly improved by the intervention after the training and they continued during the survey. I have a query on their study with special reference to job satisfaction.

By intervention, job satisfaction improved during the survey. In addition, job satisfaction in control group gradually improved and survey after 6 months showed no significant difference in the mean value between intervention group and control group. Junior gynecologists would acquire job satisfaction without intervention program and there is another process to acquire job satisfaction independent of special training content.

The trend of study outcomes after 6 months is also important. Namely, continuity of intervention effect on junior gynecologists should be clarified as further study.

\section{Compliance with ethical standards}

Conflict of interest Author declares that he has no conflict of interest.

Ethical approval This article does not contain any studies with human participation or animals performed by the author.

\section{Reference}

1. Mache S, Baresi L, Bernburg M, Vitzthum K, Groneberg D (2017) Being prepared to work in Gynecology Medicine: evaluation of an intervention to promote junior gynecologists professionalism, mental health and job satisfaction. Arch Gynecol Obstet 295:153-162
Tomoyuki Kawada

kawada@nms.ac.jp

1 Department of Hygiene and Public Health, Nippon Medical School, 1-1-5 Sendagi, Bunkyo-Ku, Tokyo 113-8602, Japan 